\title{
ARTICLE
}

\section{Germline $A T M$ variants predispose to melanoma: a joint analysis across the GenoMEL and MelaNostrum consortia}

\author{
B. Dalmasso ${ }^{1,2,31 凶}$, L. Pastorino ${ }^{1,2,31}$, V. Nathan ${ }^{3}$, N. N. Shah ${ }^{4}$, J. M. Palmer ${ }^{3}$, M. Howlie ${ }^{3}$, P. A. Johansson ${ }^{3}$, N. D. Freedman $^{4}$, \\ B. D. Carter ${ }^{5}$, L. Beane-Freeman ${ }^{4}$, B. Hicks ${ }^{6}$, A. Molven ${ }^{7,8}$, H. Helgadottir ${ }^{9}$, A. Sankar ${ }^{10}$, H. Tsao ${ }^{11}$, A. J. Stratigos ${ }^{12}$, P. Helsing ${ }^{13}$, \\ R. Van Doorn ${ }^{14}$, N. A. Gruis ${ }^{14}$, M. Visser ${ }^{14}$, K. A. W. Wadt ${ }^{15}$, G. Mann ${ }^{16}$, E. A. Holland ${ }^{16}$, E. Nagore ${ }^{17}$, M. Potrony ${ }^{18,19}$, S. Puig ${ }^{19,20}$, \\ C. Menin ${ }^{21}$, K. Peris ${ }^{22,23}$, M. C. Fargnoli ${ }^{24}$, D. Calista ${ }^{25}$, N. Soufir ${ }^{26}$, M. Harland ${ }^{27}$, T. Bishop ${ }^{27}$, P. A. Kanetsky ${ }^{28}$, D. E. Elder ${ }^{28}$, V. Andreotti ${ }^{1,2}$, \\ I. Vanni ${ }^{1,2}$, W. Bruno ${ }^{1,2}$, V. Höiom ${ }^{9}$, M. A. Tucker ${ }^{4}$, X. R. Yang ${ }^{4}$, P. A. Andresen ${ }^{29}$, D. J. Adams ${ }^{10}$, M. T. Landi ${ }^{30}$, N. K. Hayward ${ }^{3,32}$, \\ A. M. Goldstein ${ }^{4,32}$, P. Ghiorzo ${ }^{1,2,32}$, The GenoMEL* and MelaNostrum consortia*
}

PURPOSE: Ataxia-Telangiectasia Mutated (ATM) has been implicated in the risk of several cancers, but establishing a causal relationship is often challenging. Although ATM single-nucleotide polymorphisms have been linked to melanoma, few functional alleles have been identified. Therefore, ATM impact on melanoma predisposition is unclear.

METHODS: From 22 American, Australian, and European sites, we collected 2,104 familial, multiple primary (MPM), and sporadic melanoma cases who underwent ATM genotyping via panel, exome, or genome sequencing, and compared the allele frequency (AF) of selected ATM variants classified as loss-of-function (LOF) and variants of uncertain significance (VUS) between this cohort and the gnomAD non-Finnish European (NFE) data set.

RESULTS: LOF variants were more represented in our study cohort than in gnomAD NFE, both in all $(A F=0.005$ and $0.002, \mathrm{OR}=$ 2.6, $95 \% \mathrm{Cl}=1.56-4.11, p<0.01)$, and familial + MPM cases $(\mathrm{AF}=0.0054$ and $0.002, \mathrm{OR}=2.97, p<0.01)$. Similarly, VUS were enriched in all $(\mathrm{AF}=0.046$ and $0.033, \mathrm{OR}=1.41,95 \% \mathrm{Cl}=1.6-5.09, p<0.01)$ and familial $+\mathrm{MPM}$ cases $(\mathrm{AF}=0.053$ and $0.033, \mathrm{OR}=$ $1.63, p<0.01)$. In a case-control comparison of two centers that provided 1,446 controls, LOF and VUS were enriched in familial + MPM cases $(p=0.027, p=0.018)$.

CONCLUSION: This study, describing the largest multicenter melanoma cohort investigated for ATM germline variants, supports the role of ATM as a melanoma predisposition gene, with LOF variants suggesting a moderate-risk.

Genetics in Medicine (2021) 23:2087-2095; https://doi.org/10.1038/s41436-021-01240-8

\section{INTRODUCTION}

Melanoma is the deadliest form of skin cancer, with a worldwide increasing incidence and burden, especially in countries with a majority of light-skinned individuals and a high ultraviolet (UV) radiation index/exposure [1]. The etiology of melanoma is complex, resulting from the interplay of environmental, host, and hereditary factors.

Approximately $5-12 \%$ of melanomas occur in individuals with a family history of melanoma or in cancer syndrome families.
However, germline variants in known high-risk predisposition genes indicate heritability in less than half of the patients, frequently attributable to pathogenic variants in CDKN2A and, much less frequently, in CDK4. Additional genes, yet to be discovered, may also contribute to melanoma susceptibility, as has been shown for pathogenic variants of POT1, BAP1, TERT, ACD, and TERF2IP associated with less than $10 \%$ of melanomas accumulated within families [2]. More recently, other susceptibility genes such as GOLM1, EBF3, POLE, and NEK11 have been associated with

\footnotetext{
${ }^{1}$ IRCCS Ospedale Policlinico San Martino, Genetics of Rare Cancers, Genoa, Italy. ${ }^{2}$ Department of Internal Medicine and Medical Specialties, University of Genoa, Genoa, Italy. ${ }^{3}$ Oncogenomics Laboratory, QIMR Berghofer Medical Research Institute, Brisbane, QLD, Australia. ${ }^{4}$ Division of Cancer Epidemiology and Genetics, National Cancer Institute, Bethesda, MD, USA. ${ }^{5}$ American Cancer Society, Atlanta, GA, USA. ${ }^{6}$ Cancer Genomics Research Laboratory, Leidos Biomedical Research, Frederick National Laboratory for Cancer Research, Frederick, MD, USA. ${ }^{7}$ Gade Laboratory for Pathology, Department of Clinical Medicine, University of Bergen, Bergen, Norway. ${ }^{8}$ Department of Pathology, Haukeland University Hospital, Bergen, Norway. ${ }^{9}$ Department of Oncology Pathology, Karolinska Institutet and Karolinska University Hospital Solna, Stockholm, Sweden. ${ }^{10}$ Wellcome Sanger Institute, Wellcome Genome Campus, Hinxton, Cambridge, UK. ${ }^{11}$ Wellman Center for Photomedicine, Department of Dermatology, MGH Cancer Center, Massachusetts General Hospital, Boston, MA, USA. ${ }^{12}$ First Department of Dermatology-Venereology, Andreas Sygros Hospital, Medical School, National and Kapodistrian University of Athens, Athens, Greece. ${ }^{13}$ Department of Dermatology, Oslo University Hospital, Oslo, Norway. ${ }^{14}$ Department Dermatology, Leiden University Medical Center, Leiden, The Netherlands. ${ }^{15}$ Department of Clinical Genetics, University Hospital of Copenhagen, Copenhagen, Denmark. ${ }^{16}$ Centre for Cancer Research, Westmead Institute for Medical Research, University of Sydney, Westmead, Australia. ${ }^{17}$ Department of Dermatology, Instituto Valenciano de Oncologia, Valencia, Spain. ${ }^{18}$ Biochemistry and Molecular Genetics Department, Melanoma Unit, Hospital Clínic de Barcelona, IDIBAPS, Universitat de Barcelona, Barcelona, Spain. ${ }^{19}$ Centro de Investigación Biomédica en Red de Enfermedades Raras (CIBERER), Barcelona, Spain. ${ }^{20}$ Dermatology Department, Melanoma Unit, HospitalClínic de Barcelona, IDIBAPS, Universitat de Barcelona, Barcelona, Spain. ${ }^{21}$ Immunology and Molecular Oncology Unit, Veneto Institute of Oncology IOV-IRCCS, Padua, Italy. ${ }^{22}$ Institute of Dermatology, Catholic University of the Sacred Heart, Rome, Italy. ${ }^{23}$ Fondazione Policlinico Universitario A. Gemelli, IRCCS, Rome, Italy. ${ }^{24}$ Dermatology, Department of Biotechnological and Applied Clinical Sciences, University of L'Aquila, L'Aquila, Italy. ${ }^{25}$ Dermatology Unit, Maurizio Bufalini Hospital, Cesena, Italy. ${ }^{26}$ Dépatement de Génétique Moléculaire, Hôpital Bichat-Claude Bernard, Paris, France. ${ }^{27}$ Section of Epidemiology and Biostatistics, Leeds Institute of Cancer and Pathology, University of Leeds, Leeds, UK. ${ }^{28}$ Department of Cancer Epidemiology, H. Lee Moffitt Cancer Center and Research Institute, Tampa, FL, USA. ${ }^{29}$ Department of Pathology, Oslo University Hospital, Oslo, Norway. ${ }^{30}$ Divison of Cancer Epidemiology and Genetics, National Cancer Institute, National Institutes of Health, Bethesda, MD, USA. ${ }^{31}$ These authors contributed equally: B. Dalmasso, L. Pastorino. ${ }^{32}$ These authors jointly supervised this work: N. K. Hayward, A. M. Goldstein, P. Ghiorzo. *Lists of authors and their affiliations appear at the end of the paper. ${ }^{凶}$ email: brunasamia.dalmasso@dimi.unige.it
} 
Table 1. Study data set from the ten participating groups.

\begin{tabular}{|c|c|c|c|c|c|c|}
\hline Participant group ${ }^{a}$ & $\begin{array}{l}\text { Sporadic cases } \\
\text { from case-control } \\
\text { cohorts }\end{array}$ & Sequencing method & MPM cases & Sequencing method & $\begin{array}{l}\text { Familial } \\
\text { melanoma } \\
\text { probands }\end{array}$ & Sequencing method \\
\hline $\mathrm{NCl}-\mathrm{USA}$ & $783 / 11$ & Panel/ES & 3 & ES & 72 & ES \\
\hline OUH-Norway & & & 279 & ES & & \\
\hline UNIGE-Italy & 22 & Panel & 84 & Panel & 167 & Panel \\
\hline $\begin{array}{l}\text { QIMR } \\
\text { Berghofer-Australia }\end{array}$ & & & 3 & ES & 206 & ES \\
\hline $\begin{array}{l}\text { MelaNostrum/NCl- } \\
\text { USA }\end{array}$ & & & & & 201 & ES \\
\hline Sanger-UK & & & & & $90 / 25$ & $\mathrm{ES} / \mathrm{GS}$ \\
\hline KI-Sweden & & & 10 & Panel & 73 & ES \\
\hline H. Bichat-France & & & $20 / 11$ & ES/panel & $6 / 3$ & ES/panel \\
\hline MGH-USA & & & & & 29 & ES \\
\hline LUMC-Netherlands & & & & & 6 & GS \\
\hline Total & 816 & & 410 & & 878 & \\
\hline
\end{tabular}

NCI National Cancer Institute, OUH Oslo University Hospital, UNIGE Università degli Studi di Genova, QIMR Queensland Institute of Medical Research, Sanger Wellcome Sanger Institute, KI Karolinska Institute, H. Bichat Hôpital Bichat-Claude Bernard, MGH Massachussets General Hospital, LUMC Leiden University Medical Center.

${ }^{\mathrm{a}} \mathrm{See}$ Supplementary material for a complete list of the 22 contributing sites from the ten participant groups.

melanoma risk but not sufficiently validated [3]. Indeed, much of the missing heritability for melanoma may be due to the inheritance of multiple low-to-moderate risk alleles and/or shared environmental exposures that predispose to melanoma, culminating in a familial pattern of melanoma inheritance [4]. For example, several variants in MC1R, as well as MITF p.Glu318Lys, act as low/ moderate risk variants for melanoma [2]. Moreover, pathogenic variants in novel candidate genes with possible moderate risk, such as those causing oculocutaneous albinism, have been reported in melanoma families, but larger studies are needed to fully elucidate their role [5].

Melanoma can also be a subordinate cancer in the context of other multitumor cancer syndromes for which heritability is underlined by a combination of high and moderate-risk genes [4]. Some of these moderate-risk genes predispose to different types of cancers. Variant interpretation in genes with likely moderatelow risk is particularly complex because of incomplete cosegregation with the disease and incomplete penetrance. Therefore, missense substitutions in these genes are often classified as variants of uncertain significance (VUS), lacking clearly defined risk estimates.

Germline pathogenic variants in the ataxia-telangiectasia mutated (ATM) gene predispose to multiple cancers. ATM is a large (66 exon) gene [6] and has, therefore, a high number of nucleotide substitutions for primarily stochastic reasons. The ATM protein is a serine/threonine kinase involved in the DNA damage response. In particular, ATM is activated upon DNA double-strand breaks caused by ionizing radiation, oxidative stress (ROS) and, indirectly, and by DNA damage caused by UV radiation, among other functions $[6,7]$. Indeed, ATM-null cells have a high chromosomal aberrations rate and show a lack of DNA damage repair following exposure to ionizing radiation [8].

Biallelic ATM loss-of-function (LOF) variants result in ataxia-telangiectasia (AT), also known as Louis-Barr syndrome, an autosomal recessive disorder characterized by progressive cerebellar degeneration, ocular telangiectasias, immunodeficiency, and radiosensitivity, as well as predisposition to several hematologic and solid cancers. Similarly, heterozygotes for ATM variants have an increased risk of several malignancies.
For example, ATM is an established breast cancer and pancreatic cancer predisposition gene and pathogenic variants in ATM have also been implicated in susceptibility to gastric cancer and prostate cancer, suggesting that the ATM tumor spectrum is likely broad [6].

Recent studies, including the largest meta-analysis of melanoma genome-wide association studies (GWAS) to date, have linked specific low-risk variants in ATM with melanoma, although the functional alleles have not yet been determined [9]. In addition, we recently found ATM LOF or potentially deleterious variants in up to $3 \%$ of high-risk melanoma patients $[10,11]$. Therefore, we conducted a multicenter study among the GenoMEL (https:// genomel.org/) and MelaNostrum (https://dceg.cancer.gov/ research/cancer-types/melanoma/melanostrum/members) melanoma genetics consortia to further investigate the impact of ATM on the risk of developing melanoma.

\section{MATERIALS AND METHODS}

Study data sets

From centers across 22 sites of the GenoMel and MelaNostrum consortia in Europe, the United States, and Australia, we retrospectively collected information on the germline ATM status of 2,104 unrelated cutaneous melanoma cases, who were either probands from melanoma-prone families, multiple primary melanoma (MPM) cases, or melanoma cases belonging to case-control or cohort studies who tested negative for CDKN2A and CDK4 (Table 1 and Supplementary Methods). ATM genotyping was performed via panel, exome, or genome sequencing (ES/GS) at each recruiting center. In addition to ATM germline variants, each participating group was asked to provide clinical information on melanoma cases included in the study: sex, age, age at diagnosis, and personal and family history of nonmelanoma cancers. Availability of clinical information varied by contributing center. Groups also provided information on cosegregation of ATM variants in cases from melanoma-prone families, if available.

Where available, we collected similar information on 1,446 controls (individuals without a current or prior cancer diagnosis) who underwent ATM genotyping via ES (598) or panel sequencing (848). However, since controls were available for only 2 of 22 centers, we used the Genome Aggregation Database (gnomAD) [12] as the primary control group. 


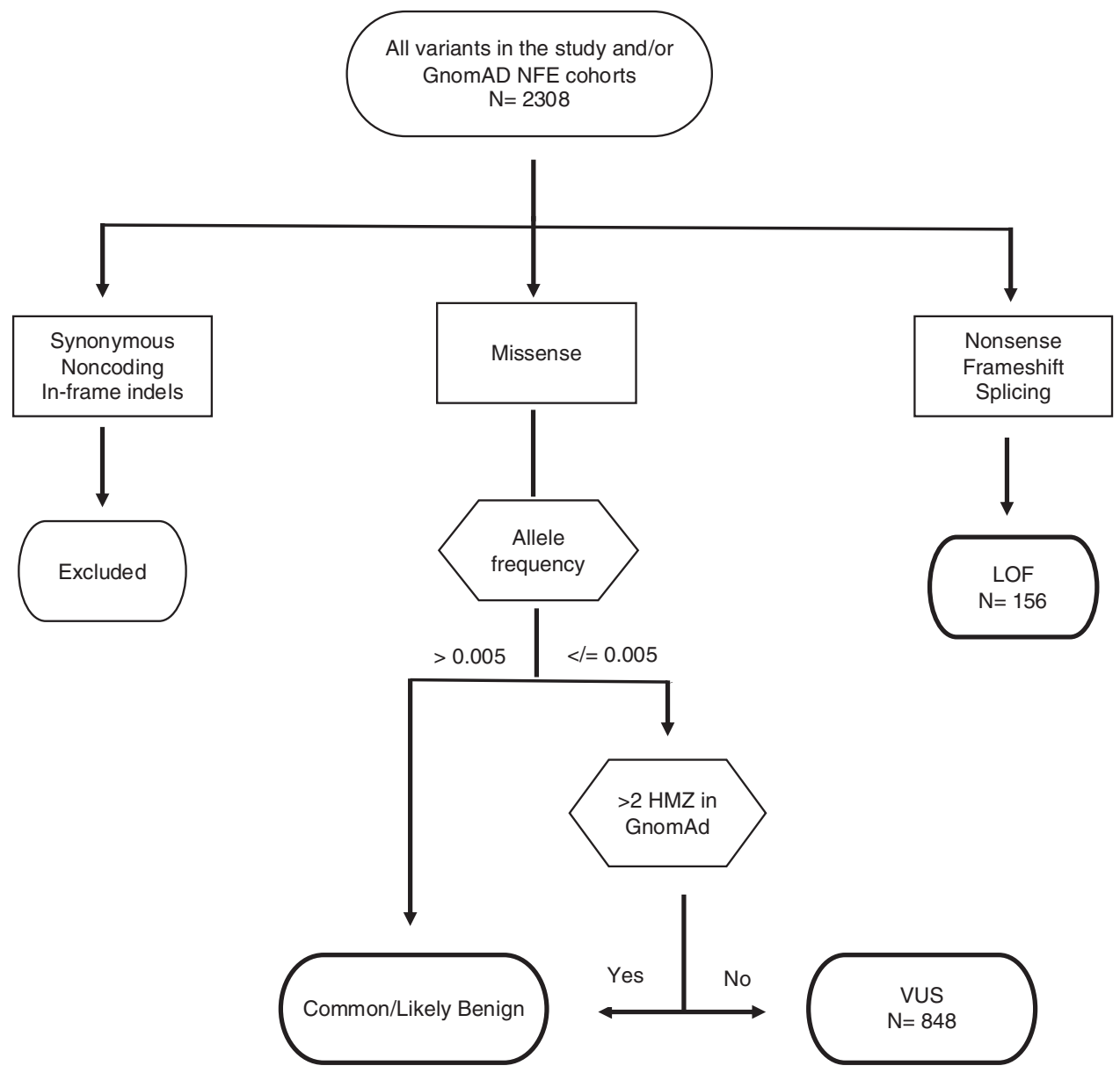

Fig. 1 ATM variants selection criteria. HMZ individuals homozygous for an ATM variant, LOF loss of function, NFE non-Finnish European, VUS variant of uncertain significance.

gnomAD version 2.1 consists of 125,748 exomes and 15,708 genomes from 141,456 germline DNA samples belonging to individuals enrolled in disease-oriented and population case-control studies. Since our study cohort included cases enrolled in countries with predominantly nonFinnish European (NFE) ancestry, we only used the gnomAD NFE subset, consisting of 64,603 individuals (56,885 exomes and 7,718 genomes). Moreover, we performed a secondary analysis comparing cases and controls from the two centers that provided controls.

\section{Variant selection and classification}

All nonsense, frameshift, splice acceptor, and splice donor ATM variants found in either our study cohort or the NFE gnomAD cohort were considered LOF and included in the analyses (ATM RefSeq NM000051.4, LRG_135 and NP000042.3).

The inclusion of missense variants was based on frequency criteria. Given that AT prevalence worldwide ranges from 1:40,000 to $1: 100,000$ [13], the estimated allele frequency of AT heterozygotes is $0.003-0.005$. Therefore, all missense variants with an allele frequency (AF) above 0.005 were considered to likely be benign and were thus excluded from the analyses. Moreover, missense variants reported to be homozygous in more than two gnomAD subjects-and thus considered benign-were also excluded. All remaining missense variants were considered VUS and included in the further analyses (Fig. 1). In addition, we reviewed PubMed indexed articles to assess each of the LOF and VUS variants found in our case cohort to evaluate their relationships to AT or cancer.

Statistical analysis and data visualization

For the purposes of our analysis, we grouped all LOF variants together and compared cases to NFE gnomAD controls. A similar approach was used for evaluating VUS. In addition, we directly examined individual variants if observed in $\geq 3$ cases.
We compared the grouped LOF variants or VUS and individual variants (in $\geq 3$ cases) in our cohort and NFE gnomAD data using Fisher's exact tests. We also conducted analyses restricting the case sample to those considered to be genetically enriched: probands from melanoma-prone families and MPM cases. For VUS only, we repeated the analysis including variants depending on whether they were located in one of the three ATM functional domains, i.e., FRAP-ATM-TRRAP (FAT), phosphatidylinositol 3kinase/phosphatidylinositol 4-kinase (PI3K/PI4K), and FAT carboxy-terminal (FATC), which encompass residues 1940-2566, 2712-2962, and 3024-3056, respectively.

In addition, we also compared grouped LOF variants or VUS in cases and controls from the two centers that provided controls.

All analyses were two-sided and a 0.05 cutoff was used for statistical significance. Statistical analyses were performed within the R computational environment [14].

The lollipop plot was generated using cBioportal Mutation Mapper $[15,16]$ and Adobe lllustrator ${ }^{\circledR}$ software.

\section{RESULTS}

Distribution of ATM variants in the study cohort compared to gnomAD

Our study cohort consisted of 2,104 cases (816 sporadic from case-control cohorts, 410 sporadic MPM, and 878 probands of melanoma-prone families). After filtering (see "Materials and Methods"), we retained 1,004 unique ATM variants, 156 LOF (12 from the study sample, 138 in NFE gnomAD, and 6 in both), and 848 unique VUS (42 in the study cohort, 731 in NFE gnomAD, and 75 in both).

Our study cohort had 20 LOF alleles in 11 familial, 3 MPM, and 6 sporadic single primary melanoma (SPM) cases $(0.95 \%$, or $1.08 \%$ 


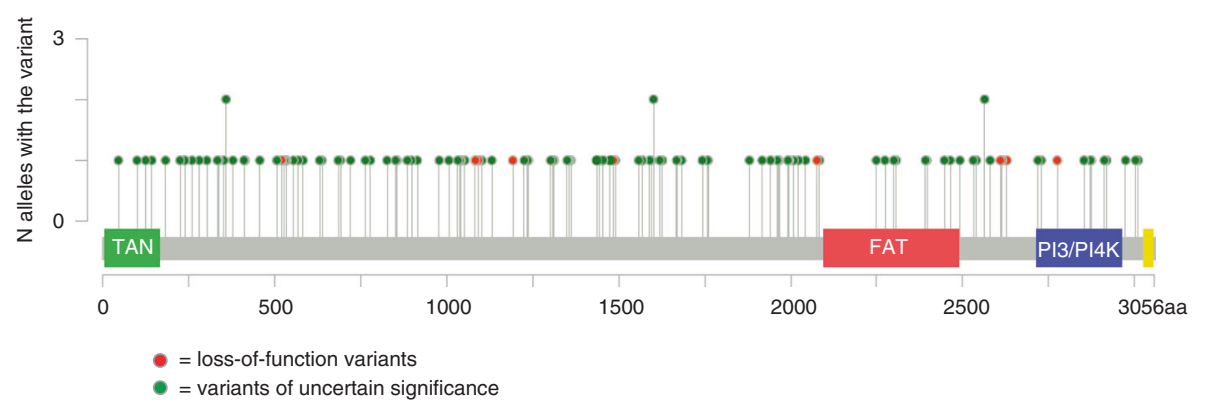

Fig. 2 The lollipop plot shows the distribution of ATM loss-of-function (LOF) variants and variants of uncertain significance (VUS) found in the study cohort. FAT FRAP-ATM-TRRAP domain, PI3/PI4K phosphatidylinositol 3-kinase/phosphatidylinositol 4-kinase-related kinase domain, TAN Tel1/ATM N-terminal motif domain.

Table 2. Frequency of ATM LOF/VUS variants in the study cohort and in the gnomAD database.

\begin{tabular}{|c|c|c|c|c|c|c|}
\hline & \multicolumn{2}{|l|}{ Study cohort } & \multicolumn{2}{|l|}{ gnomAD NFE } & \multirow[t]{2}{*}{$\mathrm{OR}^{\mathrm{a}}(95 \% \mathrm{Cl})$} & \multirow[t]{2}{*}{$p$ value } \\
\hline & $N$ variant alleles/total alleles & $\mathrm{AF}$ & $N$ variant alleles/total alleles & $\mathrm{AF}$ & & \\
\hline \multicolumn{7}{|l|}{ a. LOF } \\
\hline All & $20 / 4,208$ & 0.005 & $237 / 129,206$ & 0.002 & $2.6(1.56-4.11)$ & $2.2 \mathrm{e}-04$ \\
\hline \multicolumn{7}{|l|}{ b. VUS } \\
\hline All & $192 / 4,208$ & 0.046 & $4,268 / 129,206$ & 0.033 & $1.41(1.21-1.64)$ & $1.03 \mathrm{E}-02$ \\
\hline Fam + MPM & $135 / 2,576$ & 0.053 & & & $1.63(1.36-1.94)$ & $3.06 \mathrm{E}-04$ \\
\hline
\end{tabular}

AF allele frequency, $C l$ confidence interval, LOF loss-of-function, NFE non-Finnish European, OR odds ratio, VUS variants of uncertain significance. ${ }^{a}$ Odds of finding the variants in the study cohort compared to the odds of finding the variants in the gnomAD NFE cohort.

in the genetically enriched subset of familial + MPM cases). Moreover, there were 192 VUS alleles in 102 familial, 20 sporadic MPM, and 51 sporadic SPM cases. The distribution of LOF variants and VUS found in our study cohort across the ATM gene is shown in Fig. 2.

The NFE subset of the gnomAD cohort consists of 64,603 individuals, for a total allele count of 129,206 . We observed 4,468 alleles with an ATM variant, including 223 LOF variants and 4,245 VUS.

We observed a higher frequency of LOF variants in the study cohort compared to the NFE gnomAD controls (AF 0.005 and $0.002, \mathrm{OR}=2.6,95 \% \mathrm{Cl}=1.56-4.11, p=2.2 \mathrm{e}-04$ ), with a slightly larger difference when we restricted the analysis to the genetically enriched subset (AF 0.0054 and $0.002, \mathrm{OR}=2.97,95 \% \mathrm{Cl}=1.6$ 5.09, $p=4.9 \mathrm{e}-04$ ) (Table 2a).

One LOF variant, c.3576G >A, was observed in three unrelated Italian melanoma cases, at a frequency higher than in gnomAD NFE $(A F=0.0007$ and $0.00004, O R=20.25,95 \% \mathrm{Cl}=2.96-119.31$, $\mathrm{p}=0.001433$ ). This variant, previously thought to be a synonymous variant p.(Lys1192=), is actually a splice variant $p$. (Ser1135_Lys1195del58), resulting in the skipping of the entire exon 26 of the ATM gene [17].

Evaluation of the VUS showed a similar, albeit smaller, association, both when considering all cases (AF 0.046 and 0.033, $\mathrm{OR}=1.41,95 \% \mathrm{Cl}=1.21-1.64, p=1.033 \mathrm{e}-05)$ and the familial/MPM subset (AF 0.053 and $0.033, \mathrm{OR}=1.63,95 \% \mathrm{Cl}=$ $1.36-1.94, p=3.059 \mathrm{e}-07$ ) (Table $2 \mathrm{~b}$ ).

The association of VUS with melanoma was also present when the analysis was limited to variants located in one of the three ATM functional domains, in all cases (AF 0.013 and 0.009 , OR $=$ 1.36, $95 \% \mathrm{Cl}=1.01-1.79, p=0.0347)$ and in familial/MPM cases ( $\mathrm{AF} 0.014$ and $0.009, \mathrm{OR}=1.55,95 \% \mathrm{Cl}=1.08-2.15, p=0.0129$ ). Similar results were obtained for the subset of variants outside the
ATM functional domains: $\mathrm{AF} 0.033$ and $0.024, \mathrm{OR}=1.41, \mathrm{Cl}=$ $1.17-1.67, p=2.205 \mathrm{e}-04$ (all cases); $\mathrm{AF} 0.038$ and $0.024, \mathrm{OR}=1.63$, $\mathrm{Cl}=1.31-2, p=1.19 \mathrm{e}-05$ (familial/MPM cases).

Thirteen VUS, found in three or more cases of our study cohort, were evaluated individually (Table S1). Variant c.1368A >C p.(Leu456Phe) was only found in our study cohort. For ten variants, the allele frequency was higher in our study cohort than in gnomAD; for one of these variants, c.5750G $>C p$. (Arg1917Thr), the difference was statistically significant $(p<$ 0.05). Although, to our knowledge, there are no other studies associating C.5750G $>C$ with melanoma, this variant has been described in cancer patients. The c.5750G $>C$ variant was found in familial cases with hereditary breast and ovarian cancer enrolled in the GENESIS study cohort [18]. Two of the other VUS, c.1229T>C p.(Val410Ala) and c.6067G >A p.(Gly2023Arg), have been reported in AT patients. $[19,20]$ Moreover, c.1744T $>C$ p. (Phe582Leu), c.1229T>C, and C.6919C >T p.(Leu2307Phe) have also been previously described in individuals with hematologic malignancies [21, 22].

Of the two VUS with AF more frequent in controls, albeit not significantly $(p>0.05), c .998 \mathrm{C}>\mathrm{T}$ p.(Ser333Phe) was previously found in a child with acute leukemia who was homozygous for this variant, but without an AT diagnosis, and in a nonsyndromic homozygous colorectal cancer patient, which suggests that this variant may be benign $[22,23]$. The other variant that was more frequent in controls than cases, c.3925G $>$ A p.(Ala1309Thr), was found in 1/7,051 breast cancer cases of a Japanese case-control study cohort [24].

Additional information on all variants found in our study cohort, including previously published reports in patients with AT or cancer, as well as predictions of pathogenicity using in silico tools, is shown in Table S3. 


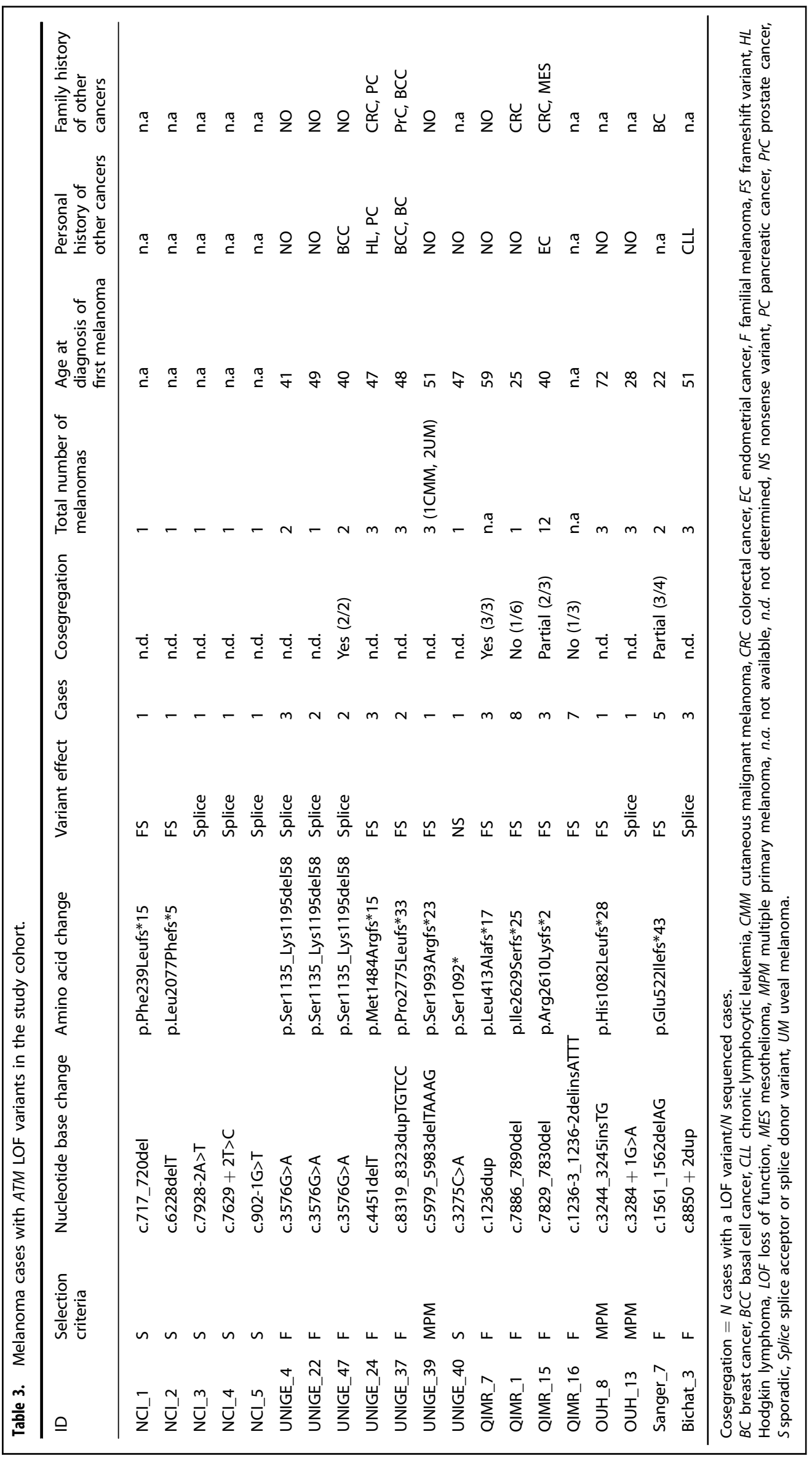


Distribution of ATM variants in cases and controls from two centers

There were 1,142 cases from the Genoa and National Cancer Institute (NCI) centers, consisting of 396 familial melanoma probands and MPM cases, plus 746 sporadic melanoma cases. The control groups, recruited by the same centers, consisted of 1,446 individuals without a current or prior history of melanoma. LOF variants and VUS found in controls are shown in Table S4.

We found ATM LOF variants in 12 cases and 6 controls (AF 0.005 and 0.002 , respectively, $\mathrm{OR}=2.54,95 \% \mathrm{Cl}=0.88-8.26, p=0.06$ ). The difference was more pronounced and significant when we restricted the analysis to the genetically enriched subset (i.e., highrisk melanoma cases), namely familial and MPM cases (AF 0.008 and $0.002, \mathrm{OR}=3.67,95 \% \mathrm{Cl}=0.98-13.77, p=0.027)$.

Overall, VUS appeared to be more frequent in controls $(\mathrm{AF}=$ 0.039 ) than in cases $(\mathrm{AF}=0.023, \mathrm{OR}=0.56,95 \% \mathrm{Cl}=0.40-0.80, p$ $=0.0008$ ); however, when the analysis was restricted to high-risk cases, the results were consistent with those of LOF variants (AF 0.060 in cases and 0.040 in controls, $\mathrm{OR}=1.54,95 \% \mathrm{Cl}=$ $1.06-2.20, p=0.018$ ).

\section{Cosegregation of ATM variants and melanoma}

ATM genotyping for affected family members with LOF variants was only available for six probands (Table 3 ). Of these, one family with two sequenced cases (UNIGE_47, c.3576G>A; p.Ser1135_Lys1195del58) and one family with three sequenced cases showed complete cosegregation of the variant (c.1236dup; p.Leu413Alafs*17) with melanoma, whereas a third family showed partial cosegregation (c.7829_7830del; p.Arg2610Lysfs*2, 2/3 cases with the variant). Similarly, family Sanger_7 showed partial cosegregation, as three of four sequenced first-degree relatives shared the same ATM variant (c.1561_1562delAG; p.Glu522llefs*43). Interestingly, although the proband had only the ATM frameshift variant, two other siblings had a concurrent CDKN2A LOF variant, whereas a fourth sibling only had a CDKN2A variant. Regardless of which of the two genes was altered at the germline level, all four individuals developed melanoma. The parents of the proband, both untested, were diagnosed with melanoma (father) and breast cancer (mother).

Conversely, in one large family, only one of six affected members sequenced had an ATM LOF variant (c.7886_7890del; p.lle2629Serfs ${ }^{*} 25$ ). However, as the affected mother of patient QIMR_1 did not carry the c.7886_7890del, this variant was either a de novo germline variant or was inherited from the unaffected father for whom no information on family history was available and, therefore, cosegregation could not be determined. Family QIMR_15 showed no evidence of cosegregation, as only one of three analyzed affected family members had the c.7829_7830del p.(Arg2610Lysfs*2) variant; however, no information was available on four additional family members who had melanoma but were not genotyped for ATM.

Information on cosegregation was available for 40 of 101 familial melanoma probands with VUS. Of these, 29 families with a single ATM variant showed evidence of cosegregation with melanoma in sequenced affected family members, and 9 probands showed partial cosegregation in their families. Among cases with two ATM variants, both variants were present in sequenced family members of NCl-Mel_2 and NCl-Mel_16 MelaNostrum families. (Table S2). Overall, most families included only two melanoma cases that were sequenced. Only 6 of 13 families with at least 3 melanoma cases sequenced showed full cosegregation.

\section{ATM variants and nonmelanoma cancers}

Nonmelanoma tumors were reported in a subset of cases and/or their families. Of the 20 probands with LOF variants, information on nonmelanoma tumors was available on 14 probands. Of these, six were diagnosed with a nonmelanoma cancer, and four of these cases also had at least one first-degree relative with a nonmelanoma cancer. Namely, the c.8850 + 2insA splice variant was found in a melanoma patient who also developed chronic lymphocytic leukemia (CLL). This variant, absent in gnomAD, has not been previously reported in AT or cancer patients. Separately, the patient harboring the c.4451delT p.(Met1484Argfs*15) variant was diagnosed with Hodgkin lymphoma and pancreatic cancer and had a positive family history of pancreatic and colorectal cancer (CRC). Moreover, c.8319_8323dupTGTCC p.(Pro2775Leufs*32) was found in a woman who was diagnosed with basal cell carcinoma (BCC) and breast cancer at age 49 and 53, respectively, and who had a first-degree relative with prostate cancer and BCC. BCC also occurred in one of the three cases with the c.3576G>A p.(Ser1135_Lys1195del58). c.1561_1562delAG p. (Glu522llefs*43) was found in a familial melanoma patient of our cohort who developed a BCC and whose mother was diagnosed with breast cancer, as reported in the previous paragraph. This variant, as well as similar deletions that cause the same frameshift with a premature stop codon, resulting in absent ATM kinase activity, have been found in AT patients [25].

The patient harboring the c.7829_7830del p.(Arg2610Lysfs*2) variant was diagnosed with endometrial cancer and had a positive family history for colorectal cancer. The c.7886_7890del p. (lle2629Serfs*25) variant was found in a patient with no other cancers other than melanoma but with a first-degree relative who developed CRC at age 61. This variant, which results in absent ATM protein expression [26], has been found in homozygous and compound heterozygous AT [26], and it has been associated with an increased breast cancer risk in heterozygotes [27]. Other ATM LOF variants were found in individuals without personal or family history of other cancers or for whom this information was not available. However, c.5979_5983delTAAAG p.(Ser1993Argfs*22), as well as c.6228delT p.(Leu2077Phefs*5), have been previously described in CLL and pancreatic cancer, respectively [28, 29]. Moreover, c.717 720del p.(Phe239Leufs*15) is a known ATM pathogenic variant found in AT patients [30]. To our knowledge, the remaining LOF variants have never been reported in the literature in relation to AT or other cancers.

Personal history of nonmelanoma cancers was reported in 32 of 110 cases with VUS for whom this information was available (29\%). Among these, the most frequent was nonmelanoma skin cancer (NMSC), which was present in 9 cases (7 BCC, 1 squamous cell carcinoma, and 1 NMSC not otherwise specified), followed by prostate cancer (6 cases), breast cancer ( 6 cases), and lymphoma ( 3 cases). Kidney, bladder, endometrial, CRC, pancreatic, thyroid, lung cancer, as well as mesothelioma, leiomyosarcoma, meningioma, glioblastoma, and ovarian teratoma were each found in one patient.

Family history could be retrieved for 77 cases with VUS. Of these, 32 cases (41\%) had at least one first-degree relative diagnosed with nonmelanoma cancers (Table S2). The most frequent cancer was pancreatic cancer, found in 8 families, followed by breast cancer ( 6 families), hematological malignancies (5 families), lung cancer, and prostate cancer (4 families each). For a complete overview of nonmelanoma cancers in our cohort, see Tables 3 and S2.

\section{DISCUSSION}

Despite the technological advances that have occurred during the last decades that led to multiple discoveries in the study of the human genome and cancer, several questions are still partially unanswered. For instance, much of the heritability of melanoma cannot be explained by germline pathogenic variants in single established melanoma predisposition gene(s). In contrast, an increased prevalence of melanoma cases has been found in known familial cancer syndromes for which an association with 
melanoma was not previously established [4]. An example of this phenomenon is exemplified by ATM, an intermediate-risk breast cancer susceptibility gene that has been implicated in melanoma susceptibility $[6,9]$. Considering that the risk of melanoma may be analogous to or even lower than that of breast cancer, collecting information on pedigrees large enough to investigate cancer predisposition is a challenge. An additional level of uncertainty is that different types of variants may confer divergent cancer risks, which can be hypothesized based on prior knowledge derived from studies on AT and high-risk breast cancer patients [31].

To our knowledge, there are no other hypothesis-driven studies investigating rare ATM variants in large international multicenter melanoma cohorts. Here, we demonstrated that ATM LOF variants are more frequent in melanoma patients than in NFE subjects in the gnomAD database, seemingly conferring moderate risk, supporting ATM as a melanoma predisposition gene. Overall, ATM LOF variants were observed in approximately $1 \%$ of the melanoma cases in our study cohort $0.95 \%$ in the whole cohort, $1.08 \%$ in FAM + MPM cases and $0.7 \%$ in sporadic cases), more than in gnomAD NFE samples $(0.36 \%)$, and approximately less than that reported for the known moderate melanoma risk variant MITF p.Glu318Lys, which ranges between $1.8 \%$ and $3.6 \%$ across different studies [32-35]. This, together with the effect estimates observed in our study, suggests that ATM might have a similar burden and act as a moderate risk gene in melanoma, similar to what has been observed in breast cancer patients [36]. However, the frequency reported here was slightly higher than reported by recent large ES and GS studies. Namely, The Cancer Genome Atlas Consortium (TCGA) found germline ATM LOF variants in only 3 of $470(0.6 \%)$ melanoma patients analyzed for a pan-cancer ES study [37]. However, in contrast to the current study sample, the TCGA study cohort was not selected based on family history of melanoma. Indeed, the majority of LOF variants in our cohort were found in high-risk melanoma patients, that is, either belonging to melanoma-prone families or diagnosed with MPM, whereas the rate of LOF variants in sporadic cases in our study is only slightly higher than that of the TCGA study cohort. Previous studies investigating ATM variants showed a higher prevalence of deleterious variants in familial and MPM cases (up to 3\%) than that of LOF variants found here. However, those estimates included missense variants that were classified as deleterious/pathogenic $[10,11]$ according to in silico prediction tools, cosegregation, and literature data, while here missense variants were classified only as VUS based on a frequency criteria (see "Materials and Methods").

The association between ATM VUS, especially those located in functional domains, and melanoma was weaker than that observed for LOF variants. One possible explanation is that, as for ATM itself, missense variants confer a lower risk compared to truncating (i.e., LOF) variants. However, although we only included rare VUS missense variants that were not found homozygous in healthy individuals, it is likely that nonpathogenic variants in this group might have diluted an association with melanoma. The fact that the subset of VUS in functional domains showed a smaller OR in our cohort compared to all VUS could also hint at the possibility that missense variants are less involved in melanoma predisposition than LOF variants. However, restricting the analysis to functional domains reduces the number of VUS and the number of individuals carrying them (both in our study cohort and in gnomAD), and therefore this difference could be simply due to the need of a larger sample size and/or to the exclusion of potentially functional variants outside the three functional domains, such as missense variants that alter protein folding. Indeed, the association with melanoma was maintained for VUS outside ATM functional domains, which may be consistent with this latter hypothesis. Thus, the reported association might have been stronger if selection based on functional assessment were possible for all missense/VUS variants.
In our cohort, personal and/or family history for ATM-related cancers, specifically breast cancer, pancreatic cancer, and hematologic malignancies, was found in 5/19 cases heterozygous for LOF variants (26\%). Although information on other tumors was only available for a subset of them, the abovementioned tumors were found in $8 / 103(7.7 \%)$ probands with VUS and in first-degree relatives of $20 / 78(25,6 \%)$ probands with VUS. Since these individuals were recruited mainly based on their personal and family history of melanoma, it is therefore unlikely that an enrichment of families with ATM-related tumors may have biased our results.

Our study design presents some limitations. The lack of availability of healthy individuals from the majority of the study groups made it impossible to perform a case-control study with in-house controls, sequenced with the same platforms and similar coverage, and, therefore, we compared our study cases against population controls from a publicly available database (gnomAD). Aware of the risk of population stratification bias due to nonoverlapping distributions of ethnic groups, we used the gnomAD NFE cohort as our control group, since the affected individuals in our study cohort either come from European countries excluding Finland, or from American and Australian centers that are composed mainly of individuals of European descent. Even with this adjustment, the possibility of population stratification bias cannot be completely excluded. However, although underpowered, a case-control analysis limited to the centers that provided ethnically matched controls showed an enrichment of ATM LOF variants and VUS in high-risk melanoma cases, consistent with the results from the main analyses.

Another limitation is that, although gnomAD is composed of data sets from several studies, it includes cancer cohorts, and, therefore, the detection of pathogenic ATM variants in this data set could be due, at least in part, to the presence of affected individuals with ATM-induced germline cancer predisposition, such as breast or pancreatic cancer cases in the TCGA data set. Therefore, the association of ATM pathogenic variants with melanoma could be higher than the one we observed. Another issue is that the case groups differed by study design, sequencing platform, and country of origin. It is not clear how this heterogeneity might have influenced the results. However, given the relatively small individual sample sizes from each group, it was not feasible to analyze individual group data separately, and therefore we analyzed all contributed data together. We also conducted subset analyses on cases purported to have the highest underlying genetic risks (familial and MPM cases). As hypothesized, we observed the strongest association in this genetically enriched subset.

If confirmed by further studies, our results could provide benefits in the clinical setting. In the era of personalized medicine, DNA damage repair genes are promising targets for novel cancer therapies. Poly (ADP-ribose) polymerase (PARP) inhibitors, for example, are used for BRCA1/2-positive breast, ovarian, and pancreatic cancer $[38,39]$ and have been FDA approved in the United States for castration-resistant ATM-deficient prostate cancer following a recent clinical trial [40]. Recent studies have also shown their potential role in the treatment of other ATMdeficient cancers [6], and clinical trials on patients with potentially actionable ATM-deficient cancers are ongoing.

The implication of ATM in melanoma development is recent, but the activation of the ATM/ATR pathway in response to UV-induced replication stress has been documented [7]. Thus, it is possible a defective activation of this pathway leads to malignant transformation and, if this is the case, ATM penetrance could be modulated by UV exposure and/or the co-occurrence of other inherited melanoma predisposing factors, such as phototype and $M C 1 R$ variants.

This study is part of a broader project aimed at fully exploring the link between ATM and melanoma. Indeed, in addition to LOF 
variants, rare missense variants were enriched in our melanoma cohort. To gain a clearer picture of the impact of ATM on melanoma, it will be necessary to carry out pathogenicity assessment of rare missense variants through functional testing. Besides its nuclear role in double-strand breaks and cell cycle checkpoint, cytoplasmic ATM plays noncanonical roles in the regulation of organelle/oxidative/energetic metabolism, which may involve melanoma biogenesis, and potentially, melanoma treatment $[41,42]$.

Moreover, an assessment of the magnitude of ATM risk in melanoma will be crucial to determine the potential clinical utility of germline ATM testing in terms of surveillance. In summary, the findings from this study support the designation of ATM as a moderate-risk melanoma susceptibility gene.

\section{DATA AVAILABILITY}

The data that support the findings of this study are available from the corresponding author upon request.

Received: 28 December 2020; Revised: 24 May 2021; Accepted: 25 May 2021;

Published online: 14 July 2021

\section{REFERENCES}

1. Whiteman DC, Green AC, Olsen CM. The growing burden of invasive melanoma: projections of incidence rates and numbers of new cases in six susceptible populations through 2031. J Invest Dermatol. 2016;136:1161-71.

2. Read J, Wadt KAW, Hayward NK. Melanoma genetics. J Med Genet. 2016;53:1-14.

3. Dalmasso B, Ghiorzo P. Evolution of approaches to identify melanoma missing heritability. Expert Rev Mol Diagn. 2020;20:523-31.

4. Leachman SA, Lucero OM, Sampson JE, Cassidy $P$, Bruno W, Queirolo $P$, et al. Identification, genetic testing, and management of hereditary melanoma. Cancer Metastasis Rev. 2017;36:77-90.

5. Nathan V, Johansson PA, Palmer JM, Howlie M, Hamilton HR, Wadt K, et al. Germline variants in oculocutaneous albinism genes and predisposition to familial cutaneous melanoma. Pigment Cell Melanoma Res. 2019;32:854-63.

6. Choi M, Kipps T, Kurzrock R. ATM mutations in cancer: therapeutic implications. Mol Cancer Ther. 2016;15:1781-91.

7. Yajima H, Lee KJ, Zhang S, Kobayashi J, Chen BPC. DNA double-strand break formation upon UV-induced replication stress activates ATM and DNA-PKcs kinases. J Mol Biol. 2009;385:800-10.

8. Kühne $M$, Riballo E, Rief N, Rothkamm K, Jeggo PA, Löbrich M. A double-strand break repair defect in ATM-deficient cells contributes to radiosensitivity. Cancer Res. 2004;64:500-8.

9. Landi MT, Bishop DT, MacGregor S, Machiela MJ, Stratigos AJ, Ghiorzo P, et al. Genome-wide association meta-analyses combining multiple risk phenotypes provide insights into the genetic architecture of cutaneous melanoma susceptibility. Nat Genet. 2020;52:494-504.

10. Goldstein AM, Xiao Y, Sampson J, Zhu B, Rotunno M, Bennett H, et al. Rare germline variants in known melanoma susceptibility genes in familial melanoma. Hum Mol Genet. 2017;26:4886-95.

11. Pastorino L, Andreotti V, Dalmasso B, Vanni I, Ciccarese G, Mandalà $M$, et al. Insights into genetic susceptibility to melanoma by gene panel testing: potential pathogenic variants in ACD, ATM, BAP1, and POT1. Cancers 2020;12:1007.

12. Karczewski KJ, Francioli LC, Tiao G, Cummings BB, Alföldi J, Wang Q, et al. The mutational constraint spectrum quantified from variation in 141,456 humans. Nature. 2020;581:434-43.

13. NIH. Ataxia-telangiectasia - Genetics Home Reference. https://ghr.nlm.nih.gov/ condition/ataxia-telangiectasia\#statistics. Accessed 2020.

14. $\mathrm{R}$ Core Team. R: A language and environment for statistical computing. $\mathrm{R}$ Foundation for Statistical Computing. 2016. https://www.r-project.org/.

15. Cerami E, Gao J, Dogrusoz U, Gross BE, Sumer SO, Aksoy BA, et al. The cBio Cancer Genomics Portal: an open platform for exploring multidimensional cancer genomics data. Cancer Discov. 2012;2:401-4.

16. Gao J, Aksoy BA, Dogrusoz U, Dresdner G, Gross B, Sumer SO, et al. Integrative analysis of complex cancer genomics and clinical profiles using the cBioPortal. Sci Signal. 2013;6:pl1.
17. Sandoval N, Platzer M, Rosenthal A, Dörk T, Bendix R, Skawran B, et al. Characterization of ATM gene mutations in 66 ataxia telangiectasia families. Hum Mol Genet. 1999;8:69-79.

18. Renault $A L$, et al. Morphology and genomic hallmarks of breast tumours developed by ATM deleterious variant carriers. Breast Cancer Res. 2018;20. https://doi. org/10.1186/s13058-018-0951-9

19. GM13805. https://www.coriell.org/0/Sections/Search/Sample_Detail.aspx?Ref= GM13805\&product=CC. Accessed August, 2020.

20. Carranza D, Vega AK, Torres-Rusillo S, Montero E, Martinez LJ, Santamaría M, et al. Molecular and functional characterization of a cohort of spanish patients with ataxia-telangiectasia. Neuromol Med. 2017;19:161-74.

21. Shieh A, Mohamed AA. A case of therapy-related acute myeloid leukemia in a patient with heterozygous mutations in the ataxia telangiectasia mutated gene. J Hematol. 2017;6:96-100.

22. Pause FG, Wacker $P$, Maillet $P$, Betts $D$, Sappino A-P. ATM gene alterations in childhood acute lymphoblastic leukemias. Hum Mutat. 2003;21:554-554.

23. Tanskanen $T$, Gylfe $A E$, Katainen $R$, Taipale $M$, Renkonen-Sinisalo $L$, Järvinen $H$, et al. Systematic search for rare variants in Finnish early-onset colorectal cancer patients. Cancer Genet. 2015;208:35-40.

24. Momozawa Y, Iwasaki Y, Parsons MT, Kamatani Y, Takahashi A, Tamura C, et al. Germline pathogenic variants of 11 breast cancer genes in 7051 Japanese patients and 11,241 controls. Nat Commun. 2018;9:1-7.

25. Driessen GJ, ljspeert $H$, Weemaes CM, Haraldsson Á, Trip $M$, Warris $A$, et al. Antibody deficiency in patients with ataxia telangiectasia is caused by disturbed B- and T-cell homeostasis and reduced immune repertoire diversity. J Allergy Clin Immunol. 2013;131:1367-1375.e9.

26. Exley AR, Buckenham S, Hodges E, Hallam R, Byrd P, Last J, et al. Premature ageing of the immune system underlies immunodeficiency in ataxia telangiectasia. Clin Immunol. 2011;140:26-36.

27. Hirotsu Y, Nakagomi H, Sakamoto I, Amemiya K, Oyama T, Mochizuki H, et al. Multigene panel analysis identified germline mutations of DNA repair genes in breast and ovarian cancer. Mol Genet Genomic Med. 2015;3:459-66.

28. Athanasakis E, Melloni E, Rigolin GM, Agnoletto C, Voltan R, Vozzi D, et al. The $p 53$ transcriptional pathway is preserved in ATMmutated and NOTCH1mutated chronic lymphocytic leukemias. Oncotarget. 2014;5:12635-45.

29. Shindo K, Yu J, Suenaga M, Fesharakizadeh S, Cho C, Macgregor-Das A, et al. Deleterious germline mutations in patients with apparently sporadic pancreatic adenocarcinoma. J Clin Oncol. 2017;35:3382-90.

30. Mosesso P, Piane M, Pepe G, Cinelli S, Chessa L. Modulation of hypersensitivity to oxidative DNA damage in ATM defective cells induced by potassium bromate by inhibition of the Poly (ADP-ribose) polymerase (PARP). Mutat Res Genet Toxicol Environ Mutagen. 2018;836:117-23.

31. Girard E, Eon-Marchais S, Olaso R, Renault AL, Damiola F, Dondon MG, et al. Familial breast cancer and DNA repair genes: Insights into known and novel susceptibility genes from the GENESIS study, and implications for multigene panel testing. Int J Cancer. 2019;144:1962-74.

32. Bertolotto C, Lesueur F, Giuliano S, Strub T, de Lichy M, Bille K, et al. A SUMOylation-defective MITF germline mutation predisposes to melanoma and renal carcinoma. Nature. 2011;480:94-98.

33. Yokoyama S, Woods SL, Boyle GM, Aoude LG, MacGregor S, Zismann V, et al. A novel recurrent mutation in MITF predisposes to familial and sporadic melanoma. Nature. 2011;480:99-103.

34. Potrony M, Puig-Butille JA, Aguilera P, Badenas C, Tell-Marti G, Carrera C, et al. Prevalence of MITF p.E318K in patients with melanoma independent of the presence of CDKN2A causative mutations. JAMA Dermatol. 2016;152:405-12.

35. Dalmasso B, Pastorino L, Ciccarese G, Andreotti V, Grillo F, Mastracci L, et al. CDKN2A germline mutations are not associated with poor survival in an Italian cohort of melanoma patients. J Am Acad Dermatol. 2019;80:1263-71.

36. Cavaciuti E, Laugé A, Janin N, Ossian K, Hall J, Stoppa-Lyonnet D, et al. Cancer risk according to type and location of ATM mutation in ataxia-telangiectasia families. Genes Chromosom Cancer. 2005;42:1-9.

37. Huang KL, Mashl RJ, Wu Y, Ritter DI, Wang J, Oh C, et al. Pathogenic germline variants in 10,389 adult cancers. Cell. 2018;173:355-370. e14

38. Tung NM, Garber JE. BRCA1/2 testing: therapeutic implications for breast cancer management. Br J Cancer. 2018;119:141-52.

39. Golan T, Hammel P, Reni M, Van Cutsem E, Macarulla T, Hall MJ, et al. Maintenance olaparib for germline BRCA -mutated metastatic pancreatic cancer. N Engl J Med. 2019;381:317-27.

40. Mateo J, Porta N, Bianchini D, McGovern U, Elliott T, Jones R, et al. Olaparib in patients with metastatic castration-resistant prostate cancer with DNA repair gene aberrations (TOPARP-B): a multicentre, open-label, randomised, phase 2 trial. Lancet Oncol. 2020;21:162-74.

41. Amirifar $P$, Ranjouri MR, Yazdani R, Abolhassani $H$, Aghamohammadi A. Ataxiatelangiectasia: a review of clinical features and molecular pathology. Pediatr Allergy Immunol. 2019;30:277-88. 
42. Najjar YG, Menk AV, Sander C, Rao U, Karunamurthy A, Bhatia R, et al. Tumor cell oxidative metabolism as a barrier to PD-1 blockade immunotherapy in melanoma. JCI Insight. 2019;4. https://doi.org/10.1172/jci.insight.124989

\section{ACKNOWLEDGEMENTS}

This work was supported by the Intramural Research Program of the National Institutes of Health (NIH), National Cancer Institute $(\mathrm{NCl})$, Division of Cancer Epidemiology and Genetics (DCEG), as well as by grants from Ministero della Salute (grant number RF-2016-02362288), Ospedale Policlinico San Martino (Ricerca Corrente), Italian Association for Cancer Research (AIRC, grant number IG15460), Lega Italiana per la Lotta contro il Cancro (LILT, 5×1000 2019 Investigator Grant), AR3 onlus, TRANSCAN grant through the Norwegian Research Council and Norwegian Cancer Society.

\section{AUTHOR CONTRIBUTIONS}

B.D., L.P., N.K.H., A.M.G., and P.G. designed and conceptualized the study and wrote the manuscript. All authors: acquisition of data, revision and final approval of the manuscript.

\section{ETHICS DECLARATION}

All subjects enrolled in this study signed an informed consent for genetic testing and genetic-based research under local Institutional Review Board (IRB) approved protocols (CE AOU San Martino Genova 10/2010; all institutions involved in human participant research received local IRB approval). All data from individuals participating in this study were de-identified.

\section{ADDITIONAL INFORMATION}

Supplementary information The online version contains supplementary material available at https://doi.org/10.1038/s41436-021-01240-8.

Correspondence and requests for materials should be addressed to B.D.

Reprints and permission information is available at http://www.nature.com/ reprints

Publisher's note Springer Nature remains neutral with regard to jurisdictional claims in published maps and institutional affiliations.

\begin{abstract}
Open Access This article is licensed under a Creative Commons Attribution 4.0 International License, which permits use, sharing, adaptation, distribution and reproduction in any medium or format, as long as you give appropriate credit to the original author(s) and the source, provide a link to the Creative Commons license, and indicate if changes were made. The images or other third party material in this article are included in the article's Creative Commons license, unless indicated otherwise in a credit line to the material. If material is not included in the article's Creative Commons license and your intended use is not permitted by statutory regulation or exceeds the permitted use, you will need to obtain permission directly from the copyright holder. To view a copy of this license, visit http://creativecommons. org/licenses/by/4.0/.
\end{abstract}

(c) The Author(s) 2021

\section{COMPETING INTERESTS}

The authors declare no competing interests.

\section{THE GENOMEL}

B. Dalmasso $\mathbb{D}^{1,2,31 凶}$, L. Pastorino ${ }^{1,2,31}$, V. Nathan ${ }^{3}$, N. N. Shah ${ }^{4}$, J. M. Palmer ${ }^{3}$, M. Howlie ${ }^{3}$, P. A. Johansson ${ }^{3}$, N. D. Freedman ${ }^{4}$, B. D. Carter ${ }^{5}$, L. Beane-Freeman ${ }^{4}$, B. Hicks ${ }^{6}$, A. Molven ${ }^{7,8}$, H. Helgadottir ${ }^{9}$, A. Sankar ${ }^{10}$, H. Tsao ${ }^{11}$, A. J. Stratigos ${ }^{12}$, P. Helsing ${ }^{13}$, R. Van Doorn ${ }^{14}$, N. A. Gruis ${ }^{14}$, M. Visser ${ }^{14}$, K. A. W. Wadt ${ }^{15}$, G. Mann ${ }^{16}$, E. A. Holland ${ }^{16}$, M. Potrony ${ }^{18,19}$, S. Puig ${ }^{19,20}$, N. Soufir ${ }^{26}$, M. Harland ${ }^{27}$, T. Bishop ${ }^{27}$, P. A. Kanetsky ${ }^{28}$, D. E. Elder ${ }^{28}$, V. Andreotti ${ }^{1,2}$, I. Vanni ${ }^{1,2}$, W. Bruno ${ }^{1,2}$, V. Höiom ${ }^{9}$, M. A. Tucker ${ }^{4}$, X. R. Yang ${ }^{4}$, P. A. Andresen ${ }^{29}$, D. J. Adams ${ }^{10}$, N. K. Hayward ${ }^{3,32}$, A. M. Goldstein ${ }^{4,32}$ and P. Ghiorzo $0^{1,2,32}$

\section{MELANOSTRUM CONSORTIA}

B. Dalmasso $\mathbb{D D}^{1,2,31 凶}$, L. Pastorino ${ }^{1,2,31}$, E. Nagore ${ }^{17}$, M. Potrony ${ }^{18,19}$, S. Puig ${ }^{19,20}$, C. Menin ${ }^{21}$, K. Peris ${ }^{22,23}$, M. C. Fargnoli ${ }^{24}$, D. Calista ${ }^{25}$, V. Andreotti ${ }^{1,2}$, I. Vanni ${ }^{1,2}$, W. Bruno ${ }^{1,2}$, M. T. Landi ${ }^{30}$ and P. Ghiorzo ${ }^{1,2,32}$ 